\title{
A. NOTE ON LATTICE OPERATIONS OF RIESZ SPACES OF ORDER BOUNDED OPERATORS
}

\author{
BORIS LAVRIC̆
}

\begin{abstract}
A.bstract. Let $L, M$ be Archimedean Riesz spaces with $M$ Dedekind complete, and let $\mathcal{L}_{b}(L, M)$ be the Riesz space of order bounded linear operators from $L$ into $M$. A theorem of Abramovic [1] on lattice operations of $\mathcal{L}_{b}(L, M)$ is generalized on Riesz spaces $L$ in which a weak form of Freudenthal's spectral theorem [4] holds.
\end{abstract}

\section{Introduction}

For terminology concerning Riesz spaces we refer the reader to [3],[5] and [8]. Let $L$ and $M$ be two Archimedean Riesz spaces. A linear operator $T: L \rightarrow M$ is said to be order bounded whenever $T$ carries order bounded subsets of $L$ onto order bounded subsets of $M$. The vector space of all order bounded operators from $L$ into $M$ is denoted by $\mathcal{L}_{b}(L, M)$. When $M$ is Dedekind complete, by a fundamental theorem of Kantorovic $\mathcal{L}_{b}(L, M)$ is likewise a Dedekind complete Riesz space (ordered by the cone of positive operators). Its lattice operations satisfy

$$
\begin{aligned}
& (S \wedge T) x=\inf \{S y+T z: y+z=x, y, z \geq 0\} \\
& (S \vee T) x=\sup \{S y+T z: y+z=x, y, z \geq 0\}
\end{aligned}
$$

for all $S, T \in \mathcal{L}_{b}(L, M)$ and each $x \in L^{+}$.

In many applications it is useful to express (under some additional conditions on $L$ ) the lattice operations of $\mathcal{L}_{b}(L, M)$ in terms of disjoint components of $x$. When $L$ has the principal projection property, Ju.A. Abramovič [1] have shown that

$$
\begin{aligned}
& (S \wedge T) x=\inf \{S y+T z: y+z=x, y \wedge z=0\} \\
& (S \vee T) x=\sup \{S y+T z: y+z=x, y \wedge z=0\}
\end{aligned}
$$

holds for all $S, T \in \mathcal{L}_{b}(L, M)$ and each $x \in L^{+}$.

Received October 16, 1989.

1985 Mathematics Subject Classification. 47B55.

Key words and phrases. Riesz space, order bounded linear operator, lattice operation, Freudenthal's
spectral theorem.

This work was supported by the Research Council of Slovenia. 
It is the purpose of our note to show that the same formulae are valid for a wider class of Riesz spaces which will be called WF-spaces. To see that WF-spaces are appropriately chosen for the generalization, we present also a partial converse of the main result.

Let $L$ be an Archimedean Riesz space. Given the element $u \in L^{+}, \mathcal{M}_{u}$ denotes the Riesz subspace of $L$ generated by the Boolean algebra $\mathcal{B}_{u}=\{v \in L: v \wedge(u-v)=0\}$ of all components of $u$. We shall say (see[4]) that $L$ is a WF-space (a weak form of Freudenthal's spectral theorem holds in $L$ ), if for each $u \in L^{+}$the subspace $\mathcal{M}_{u}$ is $u$ uniformly dense in the principal ideal $A_{u}$ generated by $u$. Recall that the well known Freudenthal's spectral theorem states that a Riesz space with the principal projection property is a WF-space.

By characterizations of WF-spaces given in [4],[7], an Archimedean Riesz space $L$ is a. WF-space if and only if the Kakutani representational space $K_{u}$ of every principal ideal $A_{u}, u \in L$, is totally disconnected. Moreover, if $L$ is an AM-space with unit $e$, and $K_{e}$ its representational space, then by $[7,2.7] L$ is a WF-space if and only if $K_{e}$ is totally disconnected.

\section{Results}

Theorem 1. Let $L$ be a WF-space and $M$ a Dedekind complete Riesz space. Then

$$
(S \wedge T) x=\inf \{S y+T z: y \wedge z=0, y+z=x\}
$$

holds for all $S, T \in \mathcal{L}_{b}(L, M)$ and each $x \in L^{+}$. An analogous formula holds for $S \vee T$.

Proof. Considering the identity $(S-S \wedge T) \wedge(T-S \wedge T)=0$ we can assume without loss of generality that $S \wedge T=0$.

Fix $x \in L^{+}$, and take an arbitrary $y \in[0, x]$. Put

$$
u=(4 y-x)^{+}, \quad v=x \wedge(2 x-4 y)^{+}
$$

and note that $u+v \geq x$. Since $L$ is a WF-space, by [4, Lemma 2,5] there exists a decomposition $x=x_{1}+x_{2}, x_{1} \wedge x_{2}=0$, such that $x_{1} \in A_{u}$ and $x_{2} \in A_{v}$. It follows from $x_{1} \in A_{u}$ that $x_{1} \wedge(4 y-x)^{-}=0$, and consequently

$$
x_{1} \leq\left(4 y-x_{2}\right)^{+} \leq 4 y
$$

By observing that $v=\left(x-(4 y-x)^{+}\right)^{+}$and $x_{2} \wedge\left(x-(4 y-x)^{+}\right)^{-}=0$ we get in a similar manner the estimate

$$
x_{2} \leq 4(x-y)
$$

Combined with the preceding inequality this shows that

$$
S x_{1}+T x_{2} \leq 4(S y+T(x-y))
$$


and by this the proof is complete.

Our next result describes the lattice operations of $\mathcal{L}_{b}(L, M)$ in terms of directed systems involving components and generalizes [6, Theorem 2.2]. Its proof goes in the same way as the proof of Corollary 1.3 of [3].

Theorem 2. Let $L$ be a WF-space and $M$ a Dedekind complete Riesz space. Then

$$
\left\{\sum_{i=1}^{n} S x_{i} \vee T x_{i}: x_{i} \wedge x_{j}=0 \text { for } i \neq j, \sum_{i=1}^{n} x_{i}=x\right\} \uparrow(S \vee T) x
$$

holds for all $S, T \in \mathcal{L}_{b}(L, M)$ and each $x \in L^{+}$. An analogous formula holds for $S \wedge T$.

A partial converse of Theorem 1 will be established for AM-spaces with unit. The details follow.

Proposition. Let $L$ be an $A M$-space with unit e, and let $M$ be a Dedekind complete Riesz space. If

$$
T^{+} x=\sup \{T y: y \wedge(x-y)=0\}
$$

holds for all $T \in \mathcal{L}_{b}(L, M)$ and each $x \in L^{+}$, then $L$ is a WF-space.

Proof. Identify in a natural way $L$ with $\mathcal{C}\left(K_{e}\right)$. It is enough to show that $K_{e}$ is totally disconnected. To this end take $t \in K_{e}$ and denote by $\delta_{t}$ the functional $\delta_{t}(x)=x(t)$, $x \in L$. If $s \in K_{e} \backslash\{t\}$, set $\varphi=\delta_{t}-\delta_{s} \in L^{\sim}$, and observe that $\varphi^{+}=\delta_{t}$. Choose now a function $x \in L^{+}$such that

$$
x(t)=2, x(s)=1 \text { and } x \geq e
$$

and note that the components of $x$ are the functions of form $x \cdot e_{W}$, where $e_{W}$ is the characteristic function of an open and closed subset $W$ of $K_{e}$.

Next, take an element $u>0$ in $M$, denote by $j: \mathbb{R} \rightarrow M$ the injective normal Riesz homomorphism $r \mapsto r u$, and let $T=j \circ \varphi$. Since

$$
\begin{aligned}
2 u & =j(x(t))=j\left(\varphi^{+}(x)\right)=T^{+} x=\sup \{T y: y \wedge(x-y)=0\} \\
& =\sup \{j \varphi y: y \wedge(x-y)=0\}=j(\sup \{\varphi(y): y \wedge(x-y)=0\}) \\
& =\sup \{\varphi(y): y \wedge(x-y)=0\} u
\end{aligned}
$$

it follows easily that $t \in W$ and $s \notin W$ for some open and closed subset $W$ of $K_{e}$. Therefore $K_{e}$ is totally disconnected as we claimed.

Remarks. An Archimedean Riesz space $L$ will be called an SF-space if any two disjoint elements of $L$ lie in two disjoint projection bands of $L$. If $L$ is an SF-space and $M$ a Dedekind complete Riesz space, the formulae from Theorem 1 can be improved as follows:

$(S \wedge T) x=\inf \{(S P+T(I-P)) x: P$ a band projection of $L\}, x \in L^{+}$and analogously for $S \vee T$.

SF-spaces are introduced and characterized in [4]. Every SF-space is a WF-space, while the Riesz space $c$ of all real convergent sequences is a WF-space which is not an SF-space. The quotient Riesz space $\ell_{\infty} / c_{0}$ is an example of an SF-space without the principal projection property. 


\section{References}

[1] J.A. Abramovič, "Injective envelopes of normed lattices," Soviet Math. Dokl. 12 (1971), 511-514.

[2] C.D. Aliprantis and O. Burkinshaw, "The components of a positive operator," Math. Z. 184 (1983), 24.5-257.

[3] C.D. Aliprantis and O. Burkinshaw, Positive Operators, Academic Press, New York (1985).

[4] B. Lavrič, "On Freudentthal's spectral theorem," Proc. Kon. Ned. Akad. Wetensch 89 (1986), $411-421$.

[5] W.A.J. Luxemburg and A.C. Zaanen, Riesz Spaces I, North-Holland, Amsterdam (1971).

[6] W.A.J. Luxemburg and A.C. Zaanen, "The linear modulus of an order bounded linear transformation I," Proc. Kon. Ned. Akad. Wetensch 74 (1971), 422-434.

[7] A.I. Veksler, "Projection properties of vector lattices and Freudenthal's spectral theorem," (Russian) Math. Nachr. 74 (1976), 7-25.

[8] A.C. Zaanen, Riesz Spaces II, North-Holland, Amsterdam (1983).

Institute of Mathematics, Physics and Mechanics, Jadranska 19, 61000 Ljubljana, Yugoslavuia. 